\title{
A Fully Fuzzy Linear Programming Model to the Berth Allocation Problem
}

\author{
Flabio Gutiérrez Segura \\ Universidad Nacional de Piura \\ Departamento de Matemáticas \\ Urb. Miraflores s/n, Perú \\ Email: flabio@unp.edu.pe
}

\author{
Edwar Luján Segura \\ Universidad Nacional de Trujillo \\ Departamento de Informática, Av. \\ Juan Pablo II s/n, Perú \\ Email: edwar_1s@hotmail.com
}

\author{
Edmundo Vergara Moreno, \\ Rafael Asmat Uceda \\ Universidad Nacional de Trujillo \\ Departamento de Matemáticas, Av. \\ Juan Pablo II s/n, Trujillo, Perú \\ Email: \{evergara, \\ rasmat $\} @$ unitru.edu.pe $\}$
}

\begin{abstract}
The berth allocation problem (BAP) in marine container terminals is defined as the feasible berth allocation to the incoming vessels. In this work, we develop a model of fully fuzzy linear programming (FFLP) for the continuous and dynamic BAP. The vessel arrival times are assumed to be imprecise, meaning that the vessel can be late or early up to a threshold permitted. Triangular fuzzy numbers represent the uncertainty of the arrivals. The model proposed has been implemented in CPLEX and evaluated for different instances. The results obtained show that the model proposed is helpful to the administrators of a marine container terminal, since a plan supporting imprecision in the arrival time of vessels, optimized with respect to the waiting time and easily adaptable to possible incidents and delays, is available to them.
\end{abstract}

\section{INTRODUCTION}

$\mathrm{I}$ $\mathrm{n}$ this work, we approach the berth allocation problem (BAP), a NP-hard problem of combinatorial optimization [1], consisting in the allocation for every incoming vessel its berthing position at the quay. Once the vessel arrives to the port, it comes a waiting time to be berthed at the quay. The administrators of Marine Container Terminal (MTC) must face with two related decisions: where and when the vessels have to be berthed.

The actual times of arrivals for each vessel are highly uncertain depending this uncertainty, for example, on the weather conditions (rains, storms), technical problems, other terminals that the vessel have to visit and other reasons. The vessels can arrive earlier or later their scheduled arrival time [2], [3]. This situation affects the operations of load and discharge, other activities at the terminal and the services required by costumers. The administrators of MTC change or reviews the plans, but a frequent review of the berthing plan is not a desirable thing from a planning of resources point of view. Therefore, the capacity of adaptation of the berthing plan is important for a good system performance that a MTC manages. As a result, a robust model providing a berthing plan that supports the possible early or lateness in the arrival time of vessels and easily adaptable is desirable.

There are many types of uncertainty such as the randomness, imprecision (ambiguity, vagueness), confusion. Many of them can be categorized as stochastic or fuzzy [4].
The fuzzy sets are specially designed to deal with imprecision.

The simulation is done in the MTC of the port of Valencia, the use of stochastic optimization models is difficult because there are no distributions of probabilities of the delays and advances of the vessels. We assume that the arrival times of vessels are imprecise, for every vessel it is necessary to request the time interval of possible arrival, as well as the more possible time the arrival occurs.

In this work, we present a model of fuzzy optimization for the continuous and dynamic BAP. This paper is organized as follows: In Section II, we present a review of literature related to the BAP under imprecision. Subsequently, in Section III, we describe the basic concepts of the work procedure. In Section IV, we propose the model of fuzzy optimization to the berth allocation problem with imprecision in the arrival of vessels. In Section V, we employ a methodology to resolve the model. In Section VI, we evaluated the model. Finally, in Section VII, we present the conclusions and future lines of research.

\section{STATE OF THE ART}

There are many attributes to classify the models related to the BAP [5]. The most important are: spatial and temporal. The spatial attribute can be discrete or continuous. In the discrete case, the quay is considered as a finite set of berths, where segments of finite length describe every berth and usually a berth just works for a vessel at once; for the continuous case, the vessels can berth at any position within the limits of the quay. The temporal attribute can be static or dynamical. In the static case, all the vessels are assumed to be at the port before performing the berthing plan; for the dynamical case, the vessels can arrive to the port at different times during the planning horizon.

In [5], the authors make an exhaustive review of the literature existing about BAP. To our knowledge, there are very few studies dealing with BAP and with imprecise (fuzzy) data.

A fuzzy MILP (Mixed Integer Lineal Programming) model for the discrete and dynamic BAP was proposed in [6]. Triangular fuzzy numbers represent the arrival times of vessels. The model and design of a method for parametric 
MILP-based solutions are presented there, but the evaluation is not shown. In the previous model, they do not address the continuous BAP. According to Bierwith [5], to design a continuous model, the planning of berthing is more complicated than for a discrete one, but the advantage is a better use of the space available at the quay.

In [7], a MILP fuzzy model for the continuous and dynamic BAP was proposed, this model assigns slacks to support possible delays or earliness of vessels but it also has an inconvenience: if a vessel arrives early or on time, the next vessel has to wait all the time considered for the possible earliness and delay. This represent a big waste of time without the use of the quay and the vessel has to stay longer than is necessary at the port.

In this work, we present and new model for the continuous and dynamic BAP that solves the problem of the previous model. This model is formulated as a fully fuzzy linear programming problem (FFLP), wherewith we obtain robust berthing plans supporting imprecision (earliness or delay) of vessels without generating unnecessary waiting times.

\section{PRELIMINARIES}

The concepts about fuzzy sets, fuzzy arithmetic and possibility distributions are taken from [8].

\section{A. Fuzzy Sets}

Definition 1. Let $X$ be the universe of discourse. A fuzzy set $\widetilde{A}$ in $X$ is a set of pairs: $\widetilde{A}=\left\{\left(x, \mu_{\widetilde{A}}(x)\right), x \in X\right\}$, where $\mu_{\widetilde{\mathrm{A}}}: \mathrm{X} \rightarrow[0,1]$ is called the membership function and $\mu_{\widetilde{\mathrm{A}}}(\mathrm{X})$ represents the degree that $x$ belongs to the set $\widetilde{\mathrm{A}}$.

In this work, we use the fuzzy sets defined on real numbers, $\mathbb{R}$. A membership function can be triangular, trapezoidal, sigmoidal, quadratic, etc. 1.

Definition 2. A fuzzy set $\widetilde{A}$ in $\mathbb{R}$ is normal if $\max _{X} \mu_{\widetilde{A}}(\mathrm{x})=$

Definition 3 . The fuzzy set $\widetilde{A}$ in $\mathbb{R}$ is convex if and only if the membership function of $\widetilde{A}$ satisfies the inequality $\mu_{\widetilde{\mathrm{A}}}\left(\beta \mathrm{x}_{1}+(1-\beta) \mathrm{x}_{2}\right) \geq \min \left[\mu_{\widetilde{\mathrm{A}}}\left(\mathrm{x}_{1}\right), \mu_{\widetilde{\mathrm{A}}}\left(\mathrm{x}_{2}\right)\right]$, $\forall x_{1}, x_{2} \in \mathbb{R}, \beta \in[0,1]$.

Definition 4. A fuzzy number is a normal and convex fuzzy set in $\mathbb{R}$.

Definition 5. A triangular fuzzy number (TFN) (see Fig. 1) is represented by $\tilde{a}=\left(a_{1}, a_{2}, a_{3}\right)$

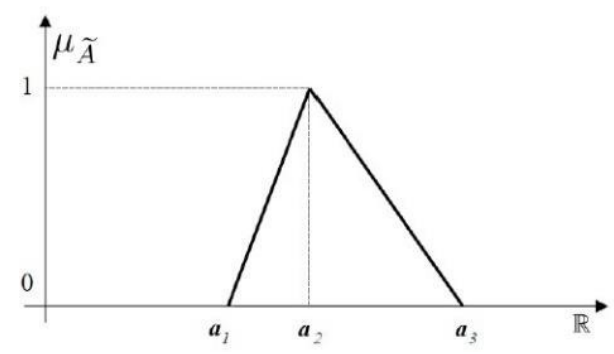

Fig. 1. Triangular fuzzy number

\section{B. Fuzzy Arithmetic}

If we have the nonnegative triangular fuzzy numbers $\tilde{a}=$ $\left(a_{1}, a_{2}, a_{3}\right)$ and $\tilde{b}=\left(b_{1}, b_{2}, b_{3}\right)$, the operations of sum and difference are defined as follows:

Sum: $\tilde{a}+\tilde{b}=\left(a_{1}+b_{1}, a_{2}+b_{2}, a_{3}+b_{3}\right)$

Difference: $\tilde{a}-\tilde{b}=\left(a_{1}-b_{3}, a_{2}-b_{2}, a_{3}-b_{1}\right)$

\section{Comparison of Fuzzy Numbers}

The comparison of fuzzy numbers allows deciding between two fuzzy numbers $\tilde{a}$ and $\tilde{b}$ which is greater, but fuzzy numbers not always provide a totally ordered set just like real numbers do. All methods for the comparison of fuzzy numbers have advantages and disadvantages.

In this work, we use the method called First Index of Yagger [9]. This method uses the ordering function

$$
\mathfrak{R}(\tilde{a})=\frac{a_{1}+a_{2}+a_{3}}{3}
$$

As a result, $\tilde{a} \leq \tilde{b}$ when $\Re(\tilde{a}) \leq \Re(\tilde{b})$, that is,

$$
a_{1}+a_{2}+a_{3} \leq b_{1}+b_{2}+b_{3}
$$

\section{Distributions of Possibility}

Imprecision can be represented by distributions of possibility [10]. These distributions allow us to formalize, in a reliable way, a very large amount of situations estimating magnitudes located in the future. The measure of possibility of an event can be interpreted as the degree of possibility of his occurrence. Among the various types of distributions, triangular and trapezoidal ones are most common. Formally, the distributions of possibility are fuzzy numbers; in this work, we use triangular distributions of possibility $\tilde{a}=$ $\left(a_{1}, a_{2}, a_{3}\right)$, which are determined by three quantities: $a_{2}$ is value with the highest possibility of occurrence, $a_{1}$ and $a_{3}$ are the upper and lower limit values allowed, respectively (see Fig. 1).

\section{E. Fully Fuzzy Linear Programming}

Fuzzy mathematical programming is useful to handle situations within optimization problems including imprecise parameters [11]. There are different approaches to the fuzzy mathematical programming. When the parameters and decision variables are fuzzy, the problem is formulated as a Fully Fuzzy Lineal Programming Problem (FFLP). There are many methodologies of solution to a FFLP [12]. Mostly of them, convert the original fuzzy model in a classical satisfactory model.

In this work, we use the method of Nasseri et. al. [13]. Given the FFLP problem

Subject to

$$
\max \sum_{j=1}^{n} \tilde{c}_{j} \tilde{x}_{j}
$$

$$
\sum_{j=1}^{n} \tilde{a}_{i j} \tilde{x}_{j} \leq \widetilde{b}_{l}, \forall i=1 \ldots m
$$


Where parameters $\tilde{c}_{j}, \tilde{a}_{i j}, \tilde{b}_{j}$ and the decision $\tilde{x}_{j}$ are nonnegative fuzzy numbers.

$$
\forall j=1 \ldots n, \quad \forall i=1 \ldots m
$$

If all parameters and decision variables are represented by triangular fuzzy numbers,

$\tilde{c}_{j}=\left(c 1_{\mathrm{j}}, c 2_{\mathrm{j}}, c 3_{\mathrm{j}}\right), \tilde{a}_{i j}=\left(a 1_{\mathrm{ij}}, a 2_{\mathrm{ij}}, \mathrm{a} 3_{i j}\right)$,

$\tilde{b}_{i}=\left(b 1_{\mathrm{i}}, b 2_{\mathrm{i}}, b 3_{\mathrm{i}}\right), \tilde{x}_{j}=\left(x 1_{\mathrm{j}}, x 2_{\mathrm{j}}, x 3_{\mathrm{j}}\right)$

Nasseri's Method transforms (1) into a classic problem of mathematical programming.

$$
\max \Re\left(\sum_{j=1}^{n}\left(c 1_{\mathrm{j}}, c 2_{\mathrm{j}}, c 3_{\mathrm{j}}\right)\left(x 1_{\mathrm{j}}, x 2_{\mathrm{j}}, x 3_{\mathrm{j}}\right)\right)
$$

Subject to:

$$
\begin{gathered}
\sum_{j=1}^{n} a 1_{i j} x 1_{i j} \leq b 1_{i}, \quad \forall i=1 \ldots m \\
\sum_{j=1}^{n} a 2_{i j} x 2_{i j} \leq b 2_{i}, \quad \forall i=1 \ldots m \\
\sum_{j=1}^{n} a 3_{i j} x 3_{i j} \leq b 3_{i}, \quad \forall i=1 \ldots m \\
x 2_{j}-x 1_{j} \geq 0, \quad x 3_{j}-x 2_{j} \geq 0, \quad \forall j=1 \ldots n
\end{gathered}
$$

Where $\mathfrak{R}$ is an ordering function (See Section III.C.)

\section{FFLP MODEL FOR THE BERTH ALLOCATION PROBLEM}

In this section, we present the notation to the main parameters used in the model (see Fig. 2).

$L$ : Total length of the quay at the MTC

$H$ : Planning horizon

Let $V$ be the set of incoming vessels, the problem data for each vessel $i \in V$ are given by:

$a_{i}$ : Arrival time at port.

$l_{i}:$ Vessel length

$h_{i}$ : Handling time of the vessel in the berth. (service time). With these data, the decision variables $m_{i}$ and $p_{i}$ must be obtained

$m_{i}$ : Berthing time of vessel.

$p_{i}$ : Berthing position where the vessel will moor.

With the data and decision variables are obtained $\omega_{i}$ and $d_{i}$

$\omega_{i}=m_{i}-a_{i}$ : Waiting time of vessel since the arrival to the berthing.

$d_{i}=m_{i}+h_{i}:$ Departure time

We consider the next assumptions: All the information related to the waiting vessels is known in advance, every vessel has a draft that is lower or equal to the draft of the quay,

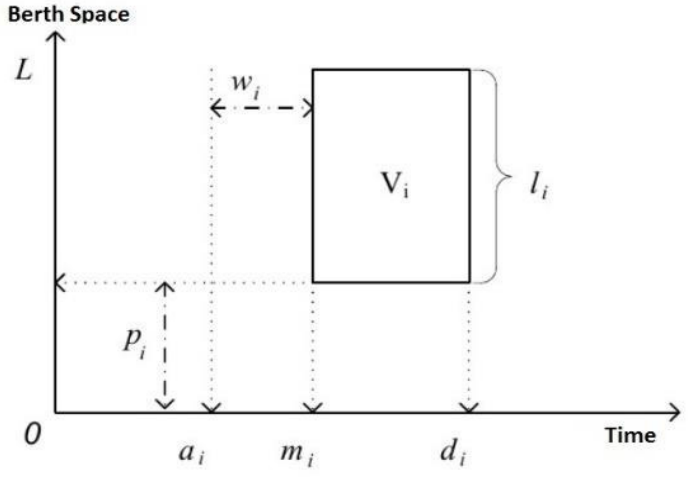

Fig. 2. Representation of a vessel according to the time and position

the berthing and departures are not time consuming, simultaneous berthing is allowed, safety distance between vessels is not considered.

The objective is to allocate all vessels according to several constraints minimizing the total waiting time, for all vessels.

The arrival times, berthing times and departure times of the vessel are considered to be of fuzzy nature (imprecise) and denoted by $\tilde{a}, \tilde{m}$, and $\tilde{h}$, respectively.

Based on the deterministic model [14] and assuming the imprecision of some parameters and decision variables, we propose the following fuzzy model optimization.

$$
\min \sum_{i \in V}\left(\tilde{m}_{i}-\tilde{a}_{i}\right)
$$

Subject to:

$\tilde{m}_{i} \geq \tilde{a}_{i} \quad \forall i \in V$

$p_{i}+l_{i} \leq L \quad \forall i \in V$

$p_{i}+l_{i} \leq p_{j}+M\left(1-z_{i j}^{x}\right) \quad \forall i, j \in V, i \neq j$

$\widetilde{m}_{i}+\tilde{h}_{i} \leq H \quad \forall i \in V$

$\tilde{m}_{i}+\tilde{h}_{i} \leq \tilde{m}_{j}+M\left(1-z_{i j}^{y}\right) \quad \forall i, j \in V, i \neq j$

$z_{i j}^{x}+z_{j i}^{x}+z_{i j}^{y}+z_{j i}^{y} \geq 1 \quad \forall i, j \in V, i \neq j$

$z_{i j}^{x}, z_{i j}^{y} \in\{0,1\} \quad \forall i, j \in V, i \neq j$

In order to assign a vessel to the quay, the following constraints must be accomplished:

(3), the berthing time of vessel must be at least the same as the arrival time; (4), there must be enough space at the quay for the berthing; (5), at the quay, a vessel must be to the left or right side of another vessel; (6), the berthing plan must be within the planning horizon; (7), with regard to the time, a vessel berths after or before another one; (8), the constraints (5) y (6) must be accomplished.

Where $z_{i j}^{x}$ is a decision variable indicating if the vessel $i$ is located to the left of vessel $\mathrm{j}$ at the berth, $\left(z_{i j}^{x}=1\right)$. 
$z_{i j}^{y}=1$ indicates that the berthing time of vessel $i$ is before than vessel $j . M$ is a large integer constant.

The planning horizon is given by

$$
H=\sum_{i \in V} h_{i}+\max _{i \in V} a_{3 i}
$$

\section{V.SOLUTION TO THE FUZZY BAP MODEL}

We assume that all the parameters and decision variables are linear and some diffuse, thus, we have a FFLP problem.

The arrival of every vessel is represented by a triangular possibility distribution $\tilde{a}=(a 1, a 2, a 3) \quad$ (see Fig. 1), indicating the possibility of arrival in $a_{2}$, but not before $a_{1}$, or after $a_{3}$. In a similar way, the berthing time is represented by $\widetilde{\mathrm{m}}=(\mathrm{m} 1, \mathrm{~m} 2, \mathrm{~m} 3)$ and $\tilde{\mathrm{h}}=(\mathrm{h}, \mathrm{h}, \mathrm{h})$ is considered a singleton.

When representing parameters and variables by triangular fuzzy numbers, we obtain a solution to the fuzzy model proposed applying the methodology proposed by Nasseri, (see section III. E).

To apply this methodology, we use the operation of fuzzy difference on the objective function and the fuzzy sum on the constraints (see Section III.B.) and the First Index of Yagger as an ordering function on the objective function (see Section III.C.) obtaining the next auxiliary MILP model.

mín $\sum_{i \in V} \frac{1}{3}\left(\left(m 1_{i}-a 3_{1}\right)+\left(m 2_{i}-a 2_{1}\right)+\left(m 3_{i}-a 1_{1}\right)\right)$

Subject to:

$$
\begin{aligned}
& m 1_{i} \geq a 1_{i} \quad \forall i \in V \\
& m 2_{i} \geq a 2_{i} \quad \forall i \in V \\
& m 3_{i} \geq a 3_{i} \quad \forall i \in V \\
& p_{i}+l_{i} \leq L \quad \forall i \in V \\
& p_{i}+l_{i} \leq p_{j}+M\left(1-z_{i j}^{x}\right) \quad \forall i, j \in V, i \neq j \\
& m 1_{i}+h_{i}+M\left(1-z_{i j}^{y}\right) \leq m 1_{j} \quad \forall i, j \in V, i \neq j \\
& m 2_{i}+h_{i}+M\left(1-z_{i j}^{y}\right) \leq m 2_{j} \quad \forall i, j \in V, i \neq j \\
& m 3_{i}+h_{i}+M\left(1-z_{i j}^{y}\right) \leq m 3_{j} \quad \forall i, j \in V, i \neq j \\
& m 2_{i}-m 1_{i}>0 \quad \forall i \in V \\
& m 3_{i}-m 2_{i}>0 \quad \forall i \in V \\
& z_{i j}^{x}+z_{j i}^{x}+z_{i j}^{y}+z_{j i}^{y} \geq 1 \quad \forall i, j \in V, i \neq j \\
& z_{i j}^{x}, z_{i j}^{y} \in\{0,1\} \quad \forall i, j \in V, i \neq j
\end{aligned}
$$

\section{EVALUATION}

The experiments were performed in 50 instances, having each of them the data arrivals for 8 vessels during a day; the instances have been generated with a uniform distribution, in order to simulate the berths at TMC of Valencia's Port (Spain). In this TMC, the quay has an approximate length of 700 meters. All the instances have the same features for all vessels (time of service and length), as well as the most possible arrival time $a_{2}$. However, all instances have different values to the minimum and maximal arrival time allowed, $a_{1}$ and $a_{3}$, respectively. The method has been coded and solved, in an optimum way, by using CPLEX. The instances were solved in a desk computer equipped with a Core (TM) i54210U CPU 2.4 Ghz with 8.00 GB RAM. The experiments were performed within a "timeout" of 60 minutes.

To report the data we use a new parameter also considered as fuzzy; the departure time of a vessel $\tilde{\mathrm{d}}=(\mathrm{d} 1, \mathrm{~d} 2, \mathrm{~d} 3)$.

One instance is shown in Table I.

TABLE I

EXAMPLE OF ONE INSTANCE

\begin{tabular}{|l|c|c|c|c|c|}
\hline \multirow{2}{*}{ Vessels } & \multicolumn{3}{|c|}{ Arrival time } & \multirow{2}{*}{} & \multirow{2}{*}{$\boldsymbol{a 1}$} \\
\cline { 2 - 4 } V1 & 4 & 8 & 34 & 121 & 159 \\
\hline V2 & 0 & 15 & 36 & 231 & 150 \\
\hline V3 & 18 & 32 & 50 & 87 & 95 \\
\hline V4 & 9 & 40 & 46 & 248 & 63 \\
\hline V5 & 32 & 52 & 72 & 213 & 219 \\
\hline V6 & 55 & 68 & 86 & 496 & 274 \\
\hline V7 & 62 & 75 & 90 & 435 & 265 \\
\hline V8 & 45 & 86 & 87 & 146 & 94 \\
\hline
\end{tabular}

For example, the most probably arrival of vessel V1 is at 8 units of time, but it could be early or late up to 4 and 34 units of time, respectively.

\begin{tabular}{|c|c|c|c|c|c|c|c|c|c|}
\hline \multirow[b]{2}{*}{ Vessel } & \multicolumn{3}{|c|}{ Berthing time } & \multirow{2}{*}{$\begin{array}{c}\begin{array}{c}\text { Service } \\
\text { t i m e }\end{array} \\
h\end{array}$} & \multicolumn{3}{|c|}{ Departure time } & \multirow[t]{2}{*}{$l$} & \multirow[t]{2}{*}{$p$} \\
\hline & $m 1$ & $m 2$ & $m 3$ & & $d 1$ & $d 2$ & $d 3$ & & \\
\hline V1 & 4 & 8 & 34 & 121 & 125 & 129 & 155 & 159 & 63 \\
\hline V2 & 0 & 15 & 36 & 231 & 231 & 246 & 267 & 150 & 222 \\
\hline V3 & 18 & 32 & 50 & 87 & 105 & 119 & 137 & 95 & 605 \\
\hline V4 & 9 & 40 & 46 & 248 & 257 & 288 & 294 & 63 & 0 \\
\hline V5 & 32 & 52 & 52 & 213 & 245 & 265 & 285 & 219 & 372 \\
\hline V6 & 245 & 265 & 265 & 496 & 741 & 761 & 781 & 274 & 332 \\
\hline V7 & 231 & 246 & 246 & 435 & 666 & 681 & 702 & 265 & 63 \\
\hline V8 & 105 & 119 & 137 & 146 & 251 & 265 & 283 & 94 & 606 \\
\hline
\end{tabular}

The berthing plan obtained with the model is showed in Table II, and polygonal-shaped are showed in Fig. 3.

The berthing plan showed in Table II provides three berthing plans. The one we could call the most optimistic assuming all the vessel arrival occurring at the minimum time allowed, is showed in columns $\mathrm{m} 1$ and $\mathrm{d} 1$ from Table II. The optimum plan, when all vessels arrive precisely on time, is given by columns $\mathrm{m} 2$ and $\mathrm{d} 2$ from Table II. The pessimistic plan assuming that all vessels are delayed the maximum allowed time is given by columns $\mathrm{m} 3$ and $\mathrm{d} 3$ from Table II.

TABLE II

BERTHING PLAN 


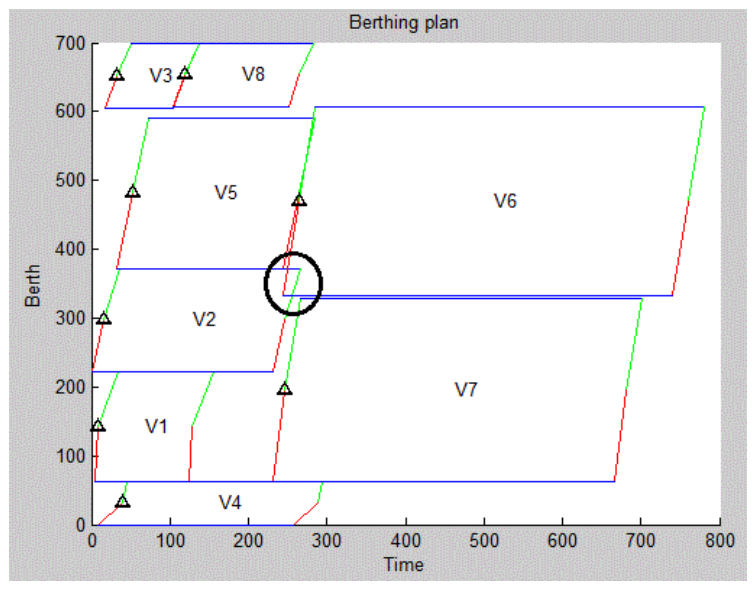

Fig. 3. Fuzzy berthing plan in polygonal-shape

An appropriate way to observe the robustness of the fuzzy berthing plan is the polygonal-shape representation (see Fig. 3 ). The red line represents the possible early Berthing time; the green line, the possible late berthing time, the small triangle represents the optimum berthing time (with a greater possibility of occurrence) and the blue line represents the time that vessel will stay at the quay.

In the circle of Fig. 3, we observe an apparent conflict between the departure of vessel V2 and the berthing of vessel V6. The conflict is not such, if the vessel V2 is late, the vessel V6 has slack times supporting delays. For example, assume that vessel V2 is late 10 units of time; according the Table II, the berthing occurs at $m=15+10=25$ units of time and its departure occurs at $\mathrm{d}=25+231=256$ units of time. The vessel V6 can moor during this space of time, since according to Table II, its berthing can occurs between 245 and 285 units of time. This fact is observed in Fig. 4.

In order to analyze the robustness of the fuzzy berthing plan, we simulate the incidences showed in Table III.

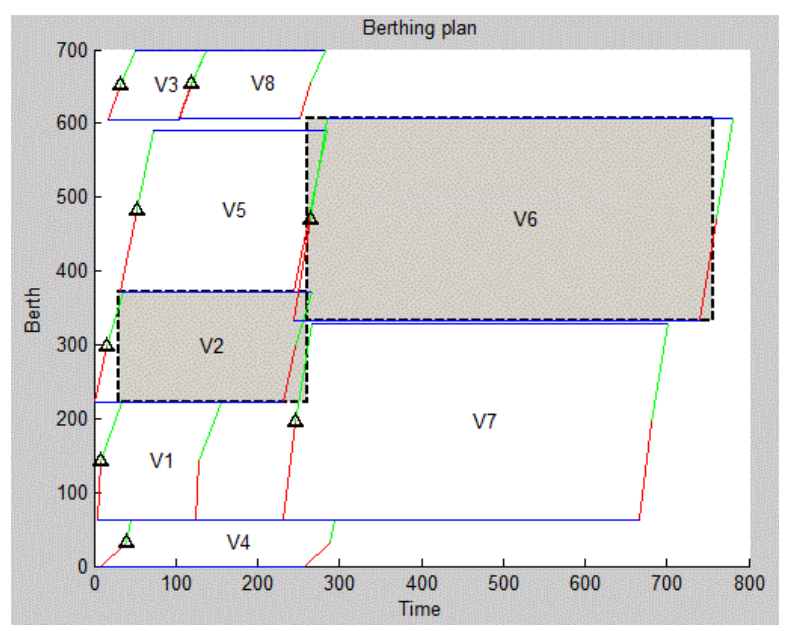

Fig. 4. Delayed berthing of vessel V2
TABLE III

INCIDENCES IN THE VESSEL ARRIVAL TIMES

\begin{tabular}{|c|c|c|}
\hline Vessel & Time & Incidence \\
\hline V1 & 13 & delay \\
\hline V2 & 15 & delay \\
\hline V3 & 0 & on time \\
\hline V4 & 18 & earliness \\
\hline V5 & 10 & earliness \\
\hline V6 & 8 & earliness \\
\hline V7 & 9 & delay \\
\hline V8 & 21 & earliness \\
\hline
\end{tabular}

To obtain a feasible and optimum berthing plan supporting the incidences, we realize a rescheduling, obtaining the berthing plan shown in Table IV. In Fig. 5, we observe that the berthing plan obtained, is a part of the fuzzy plan obtained initially.

Fig. 6 illustrates the variation of the objective function (waiting time) for 50 instances. The average of the objective function is 409.76 , that is, every day the 8 vessels have to wait a total of 409.76 units of time.

TABLE IV

BERTHING PLAN WITH RESCHEDULING

\begin{tabular}{|c|c|c|c|c|c|}
\hline Vessel & $\begin{array}{c}\text { Berthing } \\
\text { time } \\
(\boldsymbol{m})\end{array}$ & $\begin{array}{c}\text { Service } \\
\text { time } \\
(\boldsymbol{h})\end{array}$ & $\begin{array}{c}\text { Departure } \\
\text { time } \\
(\boldsymbol{d})\end{array}$ & $\begin{array}{c}\text { Length } \\
(\boldsymbol{l})\end{array}$ & $\begin{array}{c}\text { Position } \\
(\boldsymbol{p})\end{array}$ \\
\hline V1 & 21 & 121 & 142 & 159 & 63 \\
\hline V2 & 30 & 231 & 261 & 150 & 222 \\
\hline V3 & 32 & 87 & 119 & 95 & 605 \\
\hline V4 & 22 & 248 & 270 & 63 & 0 \\
\hline V5 & 42 & 213 & 255 & 219 & 372 \\
\hline V6 & 261 & 496 & 757 & 274 & 332 \\
\hline V7 & 261 & 435 & 696 & 265 & 63 \\
\hline V8 & 119 & 146 & 265 & 94 & 606 \\
\hline
\end{tabular}

On the other hand, Fig. 7, shows the computer time variation to solve the 50 instances. The average computer time that uses CPLEX to solve one instance is 2.96 seconds.

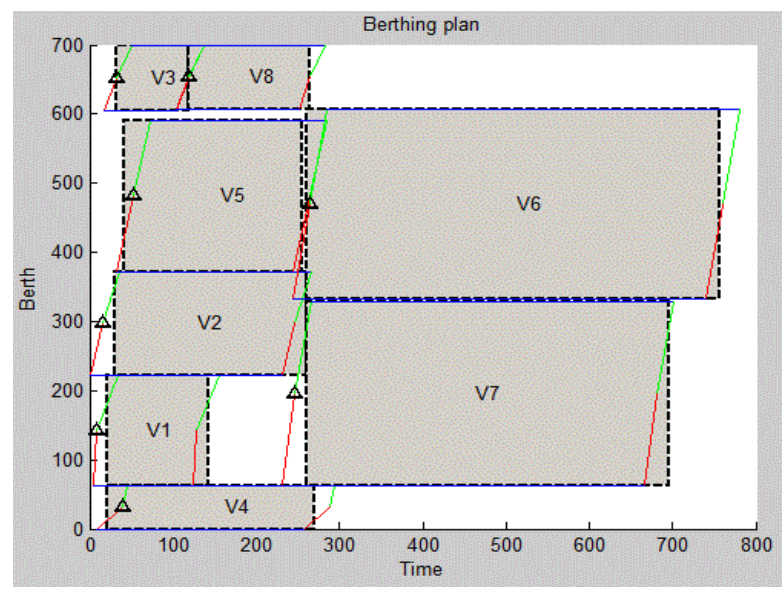

Fig. 5. Berthing with rescheduling 


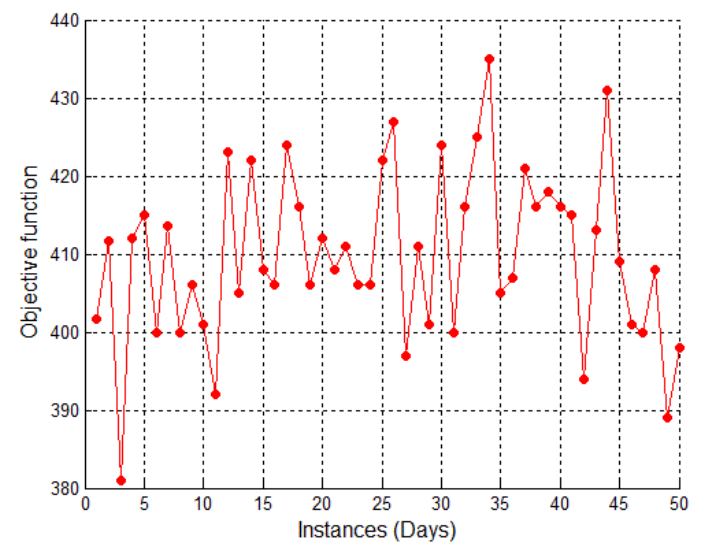

Fig. 6. Objective function for 50 instances

\section{CONCLUSION}

Even though many investigations about BAP have been carried out, most of them assume that vessel arrivals are deterministic. This is not real, in practice there are earliness or delays in vessel arrivals. Thus, the adaptability of a berthing plan is important for the global performance of the system in a MTC.

The results obtained showed that the model, is useful to the MTC managers in decision-making, since they have different plans in case the vessels arrive late, on time or early up to the maximum allowed time. In case the vessels arrive early or late a shorter time of the maximum tolerance, the optimum plan can be adapted by making a rescheduling.

The model has been evaluated for 50 instances, each consisting of 8 vessels. The number of vessel is for illustrative purposes only, the model works in the same way for a large number of vessels.

The proposed model can be used when sufficient information is not available to obtain probability distributions on the arrival time of vessels that will allow posing a stochastic model.

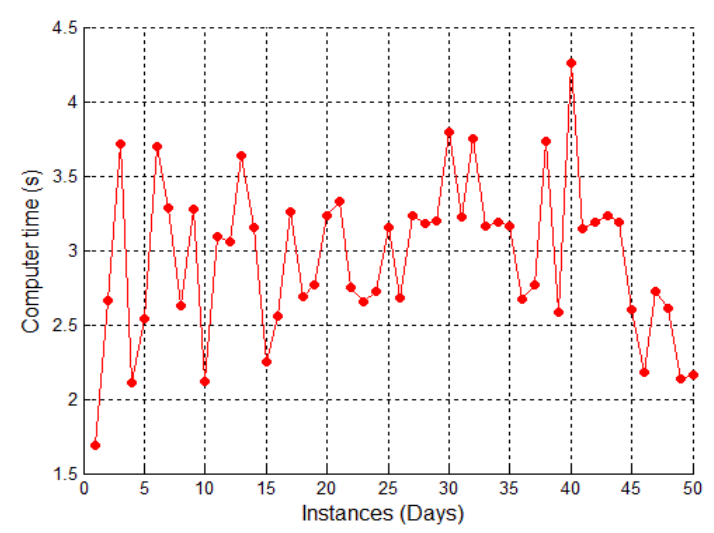

Fig. 7: Computer time of 50 instances
Likewise it could be used when we want to do berthing plans on the basis of inaccurate information obtained in advance about the vessel arrivals. For every vessel it is necessary to request the time interval of possible arrival, as well as the more possible time the arrival occurs.

Finally, because of this research, we have open problems for future researches:

- To extend the model that considers the quay cranes to be assigned to every vessel.

- To use meta-heuristics to solve the fuzzy BAP model more efficiently, when the number of vessels is greater.

\section{ACKNOWLEDGMENTS}

This work was supported by INNOVATE-PERU, Project $\mathrm{N}^{\circ}$ PIBA-2-P-069-14

\section{REFERENCES}

[1] A. Lim, "The berth planning problem", Operations Research letters, vol. 22, no 2, p. 105-110, 1998.http://dx.doi.org/10.1016/S01676377(98)00010-8

[2] M. Bruggeling, A. verbraeck, and H. Honig: "Decision support for container terminal berth planning: Integration and visualization of terminal information". In Proc. Van de Vervoers logistieke Werkdagen (VLW2011), University Press, Zelzate, p. $263-283$, 2011.

[3] M. Laumanns, et al., "Robust adaptive resource allocation in container terminals". In Proc. 25th Mini-EURO Conference Uncertainty and Robustness in Planning and Decision Making, Coimbra, Portugal, p. 501-517. 2010.

[4] H. Zimmermann, "Fuzzy set theory and its applications", Fourth Revised Edition. Springer, 2001.

[5] C. Bierwirth, F. Meisel, "A survey of berth allocation and quay crane scheduling problems in container terminals", European Journal of Operational Research, vol. 202, no 3, pp. 615-627, 2010.<http://dx.doi.org/10.1007/978-0-387-75240-2_4>

[6] B. Melián-Batista, J. Moreno-Vega, and J. Verdegay, "Una primera aproximación al problema de asignación de atraques con tiempos de llegada difusos". In. Proc. XV Congreso Español Sobre Tecnologías y Lógica Fuzzy. p. 37-42, 2010.

[7] F. Gutiérrez, E. Vergara, M. Rodríguez and F. Barber, "Un modelo de optimización difuso para el problema de atraque de barcos”. Investigación operacional, vol. 38, no. 2, pp. 160-169, 2017.

[8] L. Young-Jou, C. Hwang, "Fuzzy mathematical programming: methods and applications", vol. 394, Springer Science\& Business Media, 2012.<http://dx.doi.org/10.1007/978-3-642-48753-8>

[9] R. Yager, "A procedure for ordering fuzzy subsets of the unit interval. Information sciences", vol. 24, no.2, pp.143-161, 1981.<http://dx.doi.org/10.1016/0020-0255(81)90017-7>

[10] L. Zadeh, "Fuzzy sets as a basisfor a theory of possibility". Fuzzy sets and systems, vol. 1. no. 1, pp. 3-28.1978. <http://dx.doi.org/10.1016/0165-0114(78)90029-5>

[11] M. Luhandjula, "Mathematical programming: theory, applications and extension". Journal of Uncertain Systems, vol. 1, no. 2, 124-136. 2007.

[12] S. Das, T. Mandal, and S. Edalatpanah. "A mathematical model for solving fully fuzzy linear programming problem with trapezoidal fuzzy numbers". Applied Intelligence, pp.1-11, 2016. <http://dx.doi.org/10.1007/s10489-016-0779-x>

[13] S. Nasseri, E. Behmanesh, F. Taleshian, M. Abdolalipoor, and N. Taghi-Nezhad. "Fullyfuzzy linear programming with inequality constraints". International Journal of Industrial Mathematics, vol. 5, no. 4 , pp. 309-316, 2013.

[14] K. Kim, K. Moon. "Berth scheduling by simulated annealing," Transportation Research Part B: Methodological, vol. 37, no. 6, pp. 541-560. 2003.<http://dx.doi.org/10.1016/S0191-2615(02)00027-9> 